\title{
Predictive factors for the occurrence of idiopathic menorrhagia: Evidence for a hereditary trait
}

\author{
NATALIA KUZMINA ${ }^{1}$, JAN PALMBLAD $^{3}$ and MIRIAM MINTS ${ }^{2}$ \\ ${ }^{1}$ Department of Medicine, Division of Dermatology; ${ }^{2}$ Department of Women's and Children's Health, \\ Karolinska Institutet at Karolinska University Hospital Solna; ${ }^{3}$ Department of Medicine, Center for Inflammation and \\ Hematology Research, Karolinska Institutet at Karolinska University Hospital Huddinge, Stockholm, Sweden
}

Received April 7, 2011; Accepted May 20, 2011

DOI: $10.3892 / \mathrm{mmr} .2011 .514$

\begin{abstract}
The aim of the present study was to assess predictive factors for occurrence of idiopathic menorrhagia (IM), a disease characterized by abnormal endometrial blood vessel morphology. It was hypothesized that IM exhibits familial clustering (suggesting inheritance) and is associated with other vascular abnormalities, primarily cutaneous hemangiomas. Women with IM $(n=152)$ and healthy, regularly menstruating $(n=56)$ women answered a questionnaire concerning menstrual pattern, susceptibility to bleeding and family history of abnormal gynecological bleeding. Factor analysis with principal component extraction was used to separate predictive factors that may be associated with IM. A total of 35 different items were analyzed. A strong association was found between IM and a family history of heavy menstrual bleeding ( $\mathrm{r}=0.68)$, but not with cutaneous vascular abnormalities. Our results revealed that a family history of heavy menstrual bleeding may have the highest predictive value for the diagnosis of IM, indicating a hereditary trait.
\end{abstract}

\section{Introduction}

Heavy menstrual bleeding (HMB) of over $80 \mathrm{ml}$ blood per menstrual period (1), is common in women of reproductive age, accounting for more than $20 \%$ of outpatient gynecologic visits. Although this condition is often associated with fibroids and carcinoma, approximately $50 \%$ of $\mathrm{HMB}$ cases occur in the absence of recognized uterine pathology. Idiopathic menorrhagia (IM) (2) including regular heavy bleeding with no pelvic or uterine pathology or signs of defects in blood clotting, has been estimated to account for $9-14 \%$ of outpatient gynecologic visits (3). HMB may lead to iron deficiency

Correspondence to: Dr Miriam Mints, Department of Obstetrics and Gynecology, Karolinska University Hospital Solna, S-171 76 Stockholm, Sweden

E-mail: miriam.mints@ki.se

Key words: idiopathic menorrhagia, heavy menstrual bleeding, principal component analysis, cutaneous vascular abnormalities, heredity anemia, which requires hysterectomy. The psychological, social and employment consequences for numerous female individuals have led to the designation of $\mathrm{HMB}$ as a public health problem (4).

The etiology of IM remains largely unknown. In contrast to fibroids, for which considerable genetic influence has been shown (5), little information about possible genetic influences on IM is available. Treloar et al showed in a twin study that genetic factors accounted for $39 \%$ of the longitudinally stable variation in menstrual flow (6).

Disturbance of endometrial angiogenesis is one of several proposed underlying mechanisms of IM (7). Enhanced or aberrant angiogenesis may be expected to contribute to abnormal vascular remodeling and fragile vessels, resulting in excessive bleeding (8). In prior studies, we observed that endometrial blood vessels in IM exhibit unusual morphology, characterized both by discontinuities (gaps) in endothelial immunostaining for CD34, VWF and CD31, and by localization of pericytes on the abluminal side of these gaps. Moreover, we observed significantly greater numbers of vessels that expressed vascular endothelial growth factor (VEGF)-A, VEGF receptors (R)-1 or VEGFR-2 in IM patients than in controls. The gap size was found to correlate significantly with the vascular expression of VEGF-A and VEGFR-1 $(9,10)$. Thus, IM presents with unique morphological vascular features that may be shared with other vascular malformations, a number of which are congenital.

Congenital or inherited vascular abnormalities are classified into two major categories: hemangiomas and vascular malformations (11). Hemangiomas are benign tumors of infancy, characterized by a rapid proliferative phase followed by slow spontaneous evolution. Hemangiomas present at birth as soft, compressible, bluish or reddish tumors composed of proliferating endothelial cells. By contrast, vascular malformations are dysplastic vessels without endothelial proliferation. These malformations are identified according to the type of vessel involved (capillary, venous, arterial, lymphatic or combinations thereof) (12). The clinical picture is typical with congenital or early-developing red wine-colored plaques on skin, which disappear under diascopy (nevus flammeus). Vascular malformations may also occur in visceral organs within the respiratory and gastrointestinal tracts, but are more common in the brain. 
The etiology of vascular abnormalities is unknown, but familial predisposition is occasionally observed (13). Previous studies revealed that a mutation in the Tie- 2 gene, a receptor for angiopoietins, causes venous malformations that present as bluish-purple lesions on skin and mucous membranes (14). Investigators also suggested that endoglin gene mutations is involved in hereditary hemorrhagic telangiectasia (15).

The purpose of this study was to examine predictive factors for IM. In particular, the hypotheses of whether IM is a hereditary condition, and whether skin vascular malformations and/or hemangiomas serve as a predictive factor for IM were tested.

\section{Materials and methods}

Records for all patients diagnosed with IM between 1990 and 1999 at Danderyd's Hospital were retrieved. These female individuals underwent endometrial ablation for severe IM, where medical treatment failed to provide adequate relief of symptoms. Pre-operative blood tests revealed normal values for platelet count, activated prothrombin thromboplastin time, international normalized ratio, bleeding time and the von Willebrand factor. Pre-operatively, the uterine cavity was evaluated by hysteroscopy. These women had no discernible endometrial pathology by hysteroscopy or routine histological examination. A questionnaire was mailed (Table I) to this cohort of patients $(n=156)$. A total of 152 completed questionnaires were returned and analyzed (response rate $97 \%$ ).

Healthy controls were selected from consecutive patients who visited a gynecologist (M.M.) for routine PAP smears. Female individuals in the control group experienced a normal menstrual cycle of 24-28 days, with a 5- to 7-day duration of menstrual flow. The first 61 subjects who matched the patients were selected and received the questionnaire by mail; 56 returned completed questionnaires (response rate 92\%).

In total, 208 women received a questionnaire enquiring about menstrual pattern, susceptibility to bleeding and family history of abnormal gynecological bleeding. To clarify questions regarding vascular abnormalities, clinical images depicting nevus flammeus and hemangiomas were included in the questionnaire. The complete questionnaire is shown in Table I. The study was approved by the Ethics Committee of Karolinska University Hospital. All 208 women provided informed consent for the study.

Statistical analysis. Data were analyzed by factor analysis using principal component analysis (PCA) (16) with orthogonal rotation (varimax normalized) of the factor matrix and the Mann-Whitney U-test (Statistica 8.0; StatSoft AB, Uppsala, Sweden). This procedure (varimax normalized) ensures that the extracted factors are independent of one another and potentially reflect separate processes. The number of factors extracted for each analysis was selected using a $70 \%$ variance rule (the relevant matrix variance is accounted for when the sum of the proportionate contributions covers $>70 \%$ of the studied population and when eigenvalues were $>1.0$ ). The contribution of each behavioral variable to each factor is referred to as factor loading. The higher the loading, the more closely the variable reflects a particular factor; therefore only factor loadings $>0.5$ were included in this analysis.

\section{Results}

Demography. A total of 152 patients and 56 controls were included and the results were analyzed by principal components analysis (Table II). For all analyzed factors $(n=35)$, non-parametric analysis revealed significant $(\mathrm{p}<0.05)$ differences between the IM and control groups with respect to the occurrence of oral and fecal bleeding, family history of HMB and history of chronic drug treatment. Based on non-parametric analysis, the number of analyzed factors was reduced to 11 and included oral and intestinal bleeding, family history of HMB, use of hormonal contraceptives and other drug treatments. Finally, cumulative factor assessment of the presence of cutaneous vascular abnormalities (CVA) was also included as an independent factor, although non-parametric analysis failed to reveal differences in cutaneous vascular abnormalities between the IM and control groups. The following step was to apply PCA to determine associations between the factors referred to above and IM (presence of IM was considered to be an independent factor).

Principal components analysis. When this information was processed, five independent factors with eigenvalues $>1.0$ (Table III) were found to correspond to $74.2 \%$ of the study population $(26.3,13.2,11.8,11.6$ and $11.3 \%$ of the population, respectively).

Factor loadings are shown in Table IV and include F1, high loadings with presence of IM (0.84), family history (0.68) and history of chronic drug treatment $(0.8)$; F2, high loadings with cutaneous vascular abnormalities (0.9) and history of chronic drug treatment $(0.8)$; F3, high loadings with oral bleeding (0.67), oral contraceptives (0.70) and moderate loading of IM (0.45); and factors 4 and 5 had high loadings with variables unrelated to the topic of this study.

Score plots were then constructed using the first two extracted factors. Fig. 1 shows a clear separation between IM patients and controls along the horizontal axis (F1, highest loading of IM variability), whereas no clear separation is observed along the vertical axis (F2, highest loading of CVA).

\section{Discussion}

Regulation of menstrual blood loss in the endometrium involves a number of mechanisms. This study investigated predictive factors related to IM. The IM group included only patients with idiopathic menorrhagia, carefully selected to avoid confounding factors such as endometrial pathology and bleeding disorders, which may be hereditary.

The main observation of our study is a significant association between IM and a family history of HMB. A total of 59\% of patients in the IM group indicated such a family history compared to $14 \%$ in the control group. A significant association between chronic drug treatment and IM is understandable, since the patients were under treatment for IM.

Our findings are in concordance with, but extend beyond the limits of previous studies. In their study, Snieder et al showed that a family history is a predictive factor for hysterectomy 
Table I. Questionnaire sent to all patients and controls.

Name

Personal identity no.

Bleeding tendency, questions 1-9

Yes

Do you bruise easily (i.e., without trauma/with mild bumps?

Have you ever had bleeding in a joint or muscle?

Do your gums bleed easily (or have they done so)?

Do you bleed frequently or do you often have nosebleeds?

Have you ever noticed blood in the urine?

Have you ever coughed or vomited blood?

Have you ever had blood in the stool?

Have you ever had surgery?

If yes, what procedure(s) and when?

Did you bleed more than usual during surgery?

Have you received a blood transfusion in conjunction with surgery?

Have you ever had a tooth pulled?

Did you bleed longer than usual ( $>5 \mathrm{~h}$ ) after the procedure?

Gynecology questions

Yes

When was the first day of your last menstrual period?

How old were you when you had your first menstrual period?

Do you have or have you ever experienced heavy menstrual bleeding?

Is there a history of heavy menstrual bleeding in your immediate family (mother and/or sisters)?

How many days does your menstrual period last?

At most, how often do you change your pad/tampon?

Do you have a spiral IUD?

Do you take oral contraceptives (if yes, what kind)?

Have you used oral contraceptives in the past?

Have you ever been pregnant?

If yes:

No. of deliveries

No. of miscarriages

No. of abortions

Have you had bleeding in connection with deliveries and/or abortions, miscarriages?

Social and medical history

Yes

No

What type of work do you do?

Do you smoke? (if yes, no. of cigarettes/day)?

Do you exercise regularly? (if yes, no. of $\mathrm{x} /$ week)?

Are you overweight (weigh $>10 \mathrm{~kg} / 22 \mathrm{lb}$ over your ideal weight)?

Do you take any medications daily or at some time each week?

If yes, which medication(s)?

Have you taken any medicine for pain or fever within 10 days prior to sampling?

Have you ever taken medicine (Cyklokapron, Cyklo F, Konakion, factor concentrate)

or blood plasma at any time?

If yes, which one, and when?

Please list your past and current illnesses.

Female individuals included in the study received a questionnaire enquiring about menstrual pattern, susceptibility to bleeding and family history of abnormal gynecological bleeding. 
Table II. Results regarding occurrence of family history and $\mathrm{HMB}$ in the control group and the group with menorrhagia.

\begin{tabular}{lcc}
\hline & Mean age/years & Familial \\
\hline $\begin{array}{l}\text { Patients } \\
\text { Controls }\end{array}$ & $42.4 \pm 13$ & $59 \%(89 / 152)$ \\
$40.5 \pm 15$ & $14 \%(8 / 56)$ \\
\hline $\begin{array}{l}\text { The mean age of women with IM at the time of surgery was 42.4 years. } \\
\text { Non-parametric analysis revealed significant differences between the } \\
\text { control group and the group with IM with regards to the occurrence } \\
\text { of family history of IM (Fisher's exact test, p<0.0001). }\end{array}$ \\
\hline
\end{tabular}

Table III. Eigenvalues for the main extracted factors.

\begin{tabular}{lccc}
\hline Factor & Eigenvalue & $\begin{array}{c}\text { Total } \\
\text { variance }(\%)\end{array}$ & $\begin{array}{c}\text { Cumulative } \\
(\%)\end{array}$ \\
\hline 1 & 2.36 & 26.26 & 26.26 \\
2 & 1.19 & 13.22 & 39.48 \\
3 & 1.06 & 11.79 & 51.27 \\
4 & 1.04 & 11.61 & 62.88 \\
5 & 1.02 & 11.36 & 74.24 \\
\hline
\end{tabular}

Table IV. Factor loadings.

\begin{tabular}{|c|c|c|c|c|c|}
\hline Initial factor & Factor 1 & Factor 2 & Factor 3 & Factor 4 & Factor 5 \\
\hline Oral bleeding & 0.28 & -0.25 & 0.67 & 0.34 & -0.21 \\
\hline Faecal bleeding & 0.04 & 0.07 & 0.03 & -0.06 & -0.95 \\
\hline Gum bleeding & 0.14 & 0.12 & 0.01 & 0.88 & 0.09 \\
\hline IM & -0.84 & 0.05 & 0.45 & -0.17 & -0.01 \\
\hline Familial & 0.69 & -0.23 & -0.14 & -0.10 & -0.19 \\
\hline Contraceptive pills & -0.31 & 0.10 & 0.70 & -0.16 & 0.08 \\
\hline Drug use at the moment & 0.50 & 0.61 & 0.32 & -0.34 & 0.24 \\
\hline Prior use of tranexamic acid & $\mathbf{0 . 8 0}$ & 0.09 & 0.03 & 0.17 & 0.03 \\
\hline Skin & -0.09 & 0.90 & -0.04 & 0.13 & -0.09 \\
\hline
\end{tabular}

In general, factor loadings reflect the correlation coefficients between virtual factor and real parameter that was analyzed. Higher loadings indicate a stronger association between factor and measured parameter. Typically, loadings $<0.5$ are not considered valuable and loadings $>0.5$ are considered relevant. Loadings $>0.5$ (i.e., all relevant loadings) are marked in bold.

undertaken to control menorrhagia. The authors noted that estimates of the two main indications for hysterectomy, fibroids and menorrhagia, were similar, with heritability of 0.69 and 0.55 , respectively (17).

No data are currently available regarding the association between IM and vascular malformations of the skin or other organs. Considering that our prior results (9) showed vascular abnormalities in the endometrial blood vessels of IM patients, it is reasonable to assume that other vascular malformations may be linked to IM.

Our previous studies demonstrated specific anatomical abnormalities in endometrial blood vessels, expressed as gaps in the endothelial lining of the capillaries (9). Moreover, up-regulation of the agonist-receptor pathway of VEGF was found in IM endometrial endothelia. This deregulation of the VEGF signaling system was strongly associated with the size of the gaps. The correlation observed between family history of HMB and IM individuals may indicate the presence of polymorphism or other genetic aberrations, which play a role in the regulation of angiogenesis.

Previous studies on vascular malformations have also revealed a high frequency of autosomal dominant familial aggregation. Cerebral cavernous malformations present in the brain as dilated capillary-like vessels without tight junctions at endothelial cell interfaces, while gaps can be observed

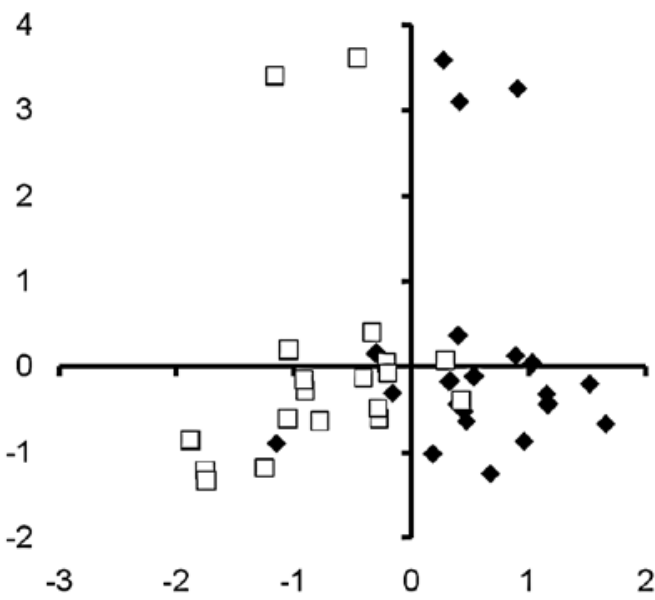

Figure 1. Score plots generated using F1 and F2 as OX and OY axes, respectively. P-group, IM patients (black rhombus); C-group, controls (white squares). The score plots were constructed using the first two extracted factors. The image shows a significant separation between IM and controls along the horizontal axis (F1), whereas no clear separation can be found along the vertical axis (F2).

between endothelial processes (14). Genetic analysis identified mutations on 7q11-22 (18).

Furthermore, previous studies have shown an association between polymorphism of the VEGF-A ${ }_{165}$ gene and diabetic 
retinopathy $(2,19)$. Awata et al showed that the genotype distribution of the $\mathrm{C}(-634) \mathrm{G}$ polymorphism differed significantly between patients with and without retinopathy (2). Our previous studies on enhanced VEGF signaling in the IM endometrium (10) and the correlation observed in this study between IM and familial HMB renders polymorphism in VEGF genes a noteworthy possibility.

The hypothesis that IM is associated with cutaneous vascular malformations was tested in the present study. Previously, it was also revealed that a mutation in the Tie-2 gene was found to cause venous malformations, present as bluish-purple lesions on skin and mucous membranes $(14,20)$. Our results did not show a significant association between IM and vascular malformations, suggesting that the etiology of IM and cutaneous vascular abnormalities is different.

In the present study, a significant difference was found between the IM and control groups with regards to the occurrence of oral and fecal bleeding, both of which are common symptoms of hemostatic diseases. Despite the fact that none of the women in the IM group met laboratory criteria for common hemostatic diseases, such as the von Willebrand factor deficiency and thrombocytopenia, other or superimposed hemostatic abnormalities cannot be excluded (21).

A correlation approach is traditionally applied to establish a link between different predictive variables and resulting variability (measure of clinical response). However, large numbers of variables render it difficult to interpret a correlation matrix. The present study employs PCA for statistical methodology. A significant advantage of this approach is that no assumptions are made regarding the distribution of data (no normalization is presumed). The only pre-processing of the data involved the scaling of each variable to a unit variance, thereby setting each variable on an equal footing. Typically, 2-3 PCA components are calculated, usually accounting for a description of $80-90 \%$ of total variance in the data, depending on data relevance. PCA scores and loadings may be projected onto one another through plots and graphs to locate data clusters and outliers. Loading plots can be used to easily determine the mannner in which each measure contributes to each factor.

In conclusion, among a number of components, only family history of HMB serves as a predictive factor for diagnosing IM. Although the mechanisms underlying IM remain poorly understood, the present data suggest a hereditary component. Previous studies have focused on molecular events and gene expression throughout the menstrual cycle (22). This study indicates that molecular endometrial genotyping provides novel opportunities for investigation and therapeutic interventions in IM.

\section{Acknowledgements}

The authors would like to express gratitude for skillful assistance with the statistical portion of this paper to Dr Alexander Kuzmin, Karolinska Institutet, Department of Clinical Neurosciences. The study was supported by grants from The Swedish Medical Research Council (19X-05991, 71XS-13135) Karolinska Institutet, Regional Agreement on Medical Training and Clinical Research (ALF) between Stockholm County Council and the Karolinska Institute, the Huddinge University Hospital and the Swedish Labor Market Insurance (AFA).

\section{References}

1. Fraser IS, Critchley HO and Munro MG: Abnormal uterine bleeding: getting our terminology straight. Curr Opin Obstet Gynecol 19: 591-595, 2007.

2. Awata T, Kurihara S, Takata N, et al: Functional VEGF C-634G polymorphism is associated with development of diabetic macular edema and correlated with macular retinal thickness in type 2 diabetes. Biochem Biophys Res Commun 333: 679-685, 2005.

3. Hallberg L, Hogdahl AM, Nilsson L and Rybo G: Menstrual blood loss and iron deficiency. Acta Med Scand 180: 639-650, 1966.

4. Gath D, Osborn M, Bungay G, et al: Psychiatric disorder and gynaecological symptoms in middle aged women: a community survey. Br Med J 294: 213-218, 1987.

5. Van Voorhis BJ, Romitti PA and Jones MP: Family history as a risk factor for development of uterine leiomyomas. Results of a pilot study. J Reprod Med 47: 663-669, 2002.

6. Treloar SA, Martin NG and Heath AC: Longitudinal genetic analysis of menstrual flow, pain, and limitation in a sample of Australian twins. Behav Genet 28: 107-116, 1998.

7. Salamonsen LA, Kovacs GT and Findlay JK: Current concepts of the mechanisms of menstruation. Baillieres Best Pract Res Clin Obstet Gynaecol 13: 161-179, 1999.

8. Krikun G, Sakkas D, Schatz F, et al: Endometrial angiopoietin expression and modulation by thrombin and steroid hormones: a mechanism for abnormal angiogenesis following long-term progestin-only contraception. Am J Pathol 164: 2101-2107, 2004.

9. Mints M, Hultenby K, Zetterberg E, et al: Wall discontinuities and increased expression of vascular endothelial growth factor-A and vascular endothelial growth factor receptors 1 and 2 in endometrial blood vessels of women with menorrhagia. Fertil Steril 88: 691-697, 2007.

10. Mints M, Blomgren B and Palmblad J: Expression of vascular endothelial growth factor receptor-3 in the endometrium in menorrhagia. Int J Mol Med 19: 909-913, 2007.

11. Mulliken JB and Glowacki J: Hemangiomas and vascular malformations in infants and children: a classification based on endothelial characteristics. Plast Reconstr Surg 69: 412-422, 1982.

12. Powell J: Update on hemangiomas and vascular malformations. Curr Opin Pediatr 11: 457-463, 1999.

13. Vikkula M, Boon LM and Mulliken JB: Molecular genetics of vascular malformations. Matrix Biol 20: 327-335, 2001.

14. Brouillard P and Vikkula M: Vascular malformations: localized defects in vascular morphogenesis. Clin Genet 63: 340-351, 2003.

15. Yamaguchi H, Azuma H, Shigekiyo T, Inoue H and Saito S: A novel missense mutation in the endoglin gene in hereditary hemorrhagic telangiectasia. Thromb Haemost 77: 243-247, 1997.

16. Joliffe IT and Morgan BJ: Principal component analysis and exploratory factor analysis. Stat Methods Med Res 1: 69-95, 1992.

17. Snieder H, MacGregor AJ and Spector TD: Genes control the cessation of a woman's reproductive life: a twin study of hysterectomy and age at menopause. J Clin Endocrinol Metab 83: 1875-1880, 1998.

18. Gunel M, Awad IA, Anson J and Lifton RP: Mapping a gene causing cerebral cavernous malformation to 7q11.2-q21. Proc Natl Acad Sci USA 92: 6620-6624, 1995.

19. Stevens A, Soden J, Brenchley PE, Ralph S and Ray DW: Haplotype analysis of the polymorphic human vascular endothelial growth factor gene promoter. Cancer Res 63: 812-816, 2003.

20. Boon LM, Mulliken JB, Vikkula M, et al: Assignment of a locus for dominantly inherited venous malformations to chromosome 9p. Hum Mol Genet 3: 1583-1587, 1994.

21. Kouides PA and Kadir RA: Menorrhagia associated with laboratory abnormalities of hemostasis: epidemiological, diagnostic and therapeutic aspects. J Thromb Haemost 5 (Suppl 1): 175-182, 2007.

22. Giudice LC: Application of functional genomics to primate endometrium: insights into biological processes. Reprod Biol Endocrinol 4 (Suppl 1): 4, 2006. 
